\title{
Utilizing public scientific web lectures to teach contemporary physics at the high school level: A case study of learning
}

\author{
Shulamit Kapon, ${ }^{1, *}$ Uri Ganiel, ${ }^{2}$ and Bat Sheva Eylon ${ }^{2}$ \\ ${ }^{1}$ The Jaime and Joan Constantiner School of Education, Tel Aviv University, Tel Aviv 69978, Israel \\ ${ }^{2}$ Department of Science Teaching, Weizmann Institute of Science, Rehovot 76100, Israel
}

(Received 19 January 2011; published 17 October 2011)

\begin{abstract}
This paper describes a teaching experiment designed to examine the learning (i.e., retention of content and conceptual development) that takes place when public scientific web lectures delivered by scientists are utilized to present advanced ideas in physics to students with a high school background in physics. The students watched an exemplary public physics web lecture that was followed by a collaborative generic activity session. The collaborative session involved a guided critical reconstruction of the main arguments in the lecture, and a processing of the key analogical explanations. Then the students watched another exemplary web lecture on a different topic. The participants $(N=14)$ were divided into two groups differing only in the order in which the lectures were presented. The students' discussions during the activities show that they were able to reason and demonstrate conceptual progress, although the physics ideas in the lectures were far beyond their level in physics. The discussions during the collaborative session contributed significantly to the students' understanding. We illustrate this point through an analysis of one of these discussions between two students on an analogical explanation of the Aharonov-Bohm effect that was presented in one of the lectures. The results from the tests that were administered to the participants several times during the intervention further support this contention.
\end{abstract}

DOI: 10.1103/PhysRevSTPER.7.020108

PACS numbers: 01.40.Fk, 01.40.gb, 01.40.Ha

\section{INTRODUCTION}

Most students will not study physics beyond the high school or undergraduate introductory level. Because the hierarchical structure of knowledge in physics makes a formal presentation of contemporary physics topics at the introductory level very difficult, most students will not be exposed to these ideas at all. This problem led us to consider the instructional use of popular presentations of contemporary physics at the high school level. In recent years many scientific programs have developed an outreach element that often includes public lectures on contemporary physics by renowned scientists. Many of these lectures are available on the web [1,2]. Although the impact of public scientific lectures on their attendees' perceptions, ideas, and opinions about scientific issues has been studied [3,4], as far as we know, no study has examined the learning of the content presented in these lectures. Extending previous studies [4-7] we thus explore ways to utilize these lectures to introduce contemporary physics at the high school level and study the learning that take place in this context.

\footnotetext{
*Corresponding author. kaponsh@post.tau.ac.il

Published by the American Physical Society under the terms of the Creative Commons Attribution 3.0 License. Further distribution of this work must maintain attribution to the author(s) and the published article's title, journal citation, and DOI.
}

Research findings indicate that teacher-centered instructional methods (e.g., lectures) are not as efficient as student-centered methods [8-10]. Hence to support and mediate learning from these web lectures, we designed generic student-centered activities as follow-ups to these lectures. The activities were aimed to help students integrate and process the content of the lecture and to be facilitated by teachers who are not necessarily specialists in the content. The activities included (1) guided note taking, (2) critical reconstruction of the arguments, and (3) processing of analogical explanations. Note taking while attending a lecture improves retention in general [11-14], but does not supply a sufficient scaffold for understanding the content delivered in a physics lecture [15]. Research on the use of argumentation in science classrooms shows that it enhances conceptual understanding [16], supports the consolidation of known ideas by making them more precise [17], and scaffolds knowledge integration [18] and, therefore, understanding $[19,20]$. The third activity was designed to examine analogies, which are a very common explanatory tool in popular scientific explanations $[5,7,21,22]$, but can also lead to the generation of misconceptions [23-28]. Some studies suggest that students benefit from explicating the correspondence between the analog objects and their relationships [29,30]. Engaging students in active criticism of the analogy and an evaluation of its limitations can also enhance their understanding [25,29,31]. We engaged our students in both activities. 
This paper describes a case study drawn from a teaching experiment that was designed to study the learning that take place when public physics lectures are utilized to teach contemporary physics at the high school level. Specifically we analyzed students' discussion of an analogy to everyday phenomena which explains the Aharonov-Bohm (AB) effect [32] in a popular manner. The explanation of the effect that appears in most textbooks [33] requires an advanced knowledge in physics, which the students did not have. The analysis illustrates the conceptual progress that the students went through during the discussion although the formal explanation of this phenomenon was clearly above their level in physics. We use the results of the tests that were administered during and after the intervention to further support our contention that meaningful learning took place.

\section{METHOD}

\section{A. Sample}

Fourteen students who had just graduated from a college preparatory physics course in physics and mathematics took part in this experiment. The course covered advanced high school mathematics and physics and was specifically designed to prepare young adults who did not take these courses in high school for university entrance exams. The physics course was algebra based and covered high school level classical mechanics, electricity, and magnetism, and some geometric optics, physical optics, and modern physics. The experiment took place about two weeks after the final exam, and the participants were paid for their time (2 days). They were divided into two groups that were very similar in level (group 1 and group 2) according to their psychometric test scores (equivalent to the SAT) and their final grades in mathematics and physics in the preacademic course.

\section{B. Lectures}

Two exemplary public physics web lectures were used: one in the domain of astrophysics and one in the domain of quantum mechanics (QM). An analysis of these lectures appears elsewhere and shows that they shared the same explanatory framework $[4,7]$.

\section{Activities accompanying the Lectures}

1. Guided note taking.-Each student watched the web lecture on a separate computer with headphones and was able to stop the lecture at any time to take notes. The students filled in an individual note-taking worksheet while watching the lecture.

2. Critical reconstruction of the scientific arguments.This activity was previously used with in-service high school physics teachers. It is structured in an individualsmall group-class sequence and is described in detail elsewhere [6]. The Appendix includes a short summary of this activity.

3. Regeneration of analogical inferences. - This activity is structured in an individual-small group-class sequence. The students are required to explicate the correspondence between the analog objects and their relationships, criticize the analogy, and evaluate its limitations. The Appendix describes this activity in detail.

\section{Procedure}

Both groups watched a lecture, engaged in a collaborative session about this lecture, and then watched the second lecture. The difference between the groups was the order in which the lectures were presented. Iterative tests were administered at several points in time during the intervention (see the Appendix). All the pair and triad discussions during the collaborative session were audiotaped, and the class discussions were videotaped. Table I summarizes this procedure.

A qualitative analysis was carried out on the transcript of one pair of students throughout the whole intervention. The pair worked on the quantum mechanics lecture and was chosen for two reasons. First, their engagement was noticeable, and during their discussion they made impressive conceptual progress that they successfully shared later with the class. Second, although all the pair and triad discussions were audiotaped, the sound quality in some was so low that a good transcription of these audiotapes was not possible. We used the class discussions and the results from the tests administered to both groups for further triangulation.

The reliability of the assessment of the open-ended questions was examined by comparing the inter-rater agreement on $30 \%$ of the tests ( $90 \%$ agreement). The validity was examined by a partial repetition of the study, involving

TABLE I. Procedure.

\begin{tabular}{lc}
\hline \hline Tests & Content of tests (Who took the test) \\
\hline $\begin{array}{l}\text { 1. Pretest } \\
\text { 2. Postwatching I: Posttest after watching the first lecture. }\end{array}$ & Lecture A and Lecture B (group 1 and Group 2) \\
Students' summary available. & Lecture A (group 1); Lecture B (group 2) \\
$\begin{array}{l}\text { 3. Poststudying: Posttest after the collaborative activity. No summary available. } \\
\text { 4. Postwatching II: Posttest after watching the second lecture. }\end{array}$ & Lecture A (group 1); Lecture B (group 2) \\
$\begin{array}{l}\text { Students' summary available. } \\
\text { 5. Post-delayed: Delayed posttest after a year. No summary available. }\end{array}$ & Lecture B (group 1); Lecture A (group 2) \\
\hline \hline
\end{tabular}


only one lecture, and the scores from a pretest and a posttest administered immediately after viewing. It was conducted a year later with different students $(N=9)$ in the same phase of their studies as the initial groups. The test results from this partial repetition were similar to those of the initial study (Pretest: $M_{\text {group } 1}=1.2, \mathrm{STD}_{\text {group } 1}=2.5$; $M_{\text {group 3 }}=2.3, \mathrm{STD}_{\text {group 3 }}=3$. Postwatching I: $M_{\text {group 1 }}=$ $37.7, \mathrm{STD}_{\text {group } 1}=16.5 ; M_{\text {group } 3}=38.0, \mathrm{STD}_{\text {group } 3}=$ 14.0).

\section{RESULTS}

\section{A. Students discussing the Aharonov-Bohm effect}

This section presents excerpts from one of the discussions that was conducted in small groups during the collaborative session that followed the QM lecture. The discussants are a pair of students, Ran and Gal (pseudonyms). The Appendix presents a summary of the QM lecture and the activities for the whole collaborative session that processed this lecture. The following discussion took place while the students were working together on a worksheet that asked them to rethink the analogical explanation of the Aharonov-Bohm effect presented in the lecture (see Fig. 1). The Appendix presents the worksheet that initiated this discussion.

Both students mapped the source onto the target successfully (see the Appendix), identifying the relevant components and the common structure. They did this quickly and expressed no difficulty. However, their subsequent discussion initiated by the next question in the worksheet"Why is the electron in the conducting loop of Aharonov-Bohm experiment affected by the magnetic field inside the loop? - revealed their difficulties with the analogical inference.

(a) The limitations of the analogy

445. Gal: It's not as though this electron communicates with that electron.

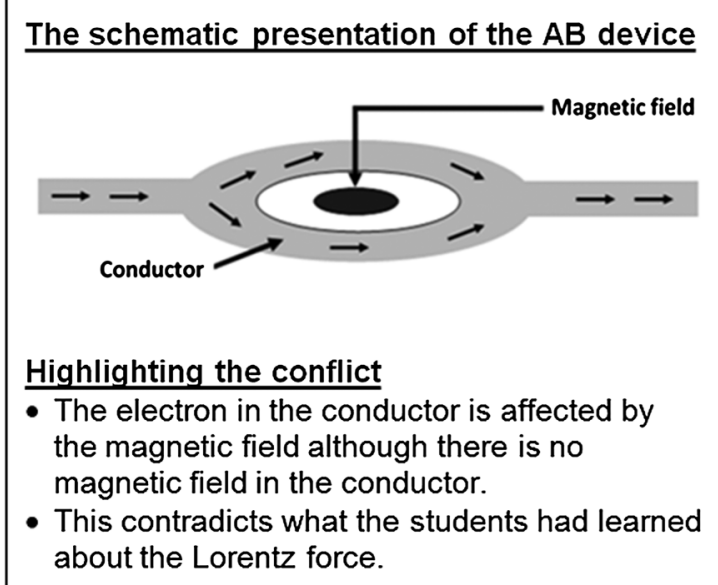

446. Ran: It is. It's the same electron...

447. Gal: But how does it communicate [laughs]?

448. Ran: It's the same electron.

449. Gal: But it's not like a person who is connected, and the brain understands it.

450 Ran: It is. I don't know, according to the theory, it is...

In the above episode Gal erroneously represents the electron as being divided into two partial electrons, each moving in a different branch, such that these partial entities communicate in some mysterious way. Perhaps Gal's difficulties stem from the limitation of the analogy. A person cannot really be in two places at the same time simultaneously, one foot is on the road and one is on the sidewalk. The brain uses this simultaneous information to infer height differences. Gal is looking for the "brain" function in the electron. However there is no "brain" equivalent in the electron. Gal still perceives the electron as a particle and thus finds it hard to accept that the electron is not divided into two separate particles that communicate, and that the whole electron is in both places at the same time.

(b) In search of a causal mechanism

451. Gal: There must be more to it. Let's say that we have a disturbance that spreads out simultaneously, OK? There is a wave. What does it do when it bumps into an obstacle? It bypasses it [diffraction]. OK? So maybe when it bypasses it... [pause]

Gal, dissatisfied with the analogical explanation, started to think about waves. This could have been prompted by the lecture. The phrase "disturbance that spreads out simultaneously" as a description of a wave was specifically mentioned in the lecture; the claim that the $\mathrm{AB}$ effect manifests the wave property of electrons was repeated several times. Gal then also began to recall what he knew about waves, and what seemed relevant to the phenomenon

\section{Explaining via an analogy}

- A person who has one foot on the road and one foot on the sidewalk can simultaneously feel the height difference between the road and the sidewalk.

- If this person stood with both feet on the sidewalk or both feet on the road, she could not detect the difference in height. Thus standing on the road and the sidewalk simultaneously allows the person to "feel" the height difference between the two.

- Similarly, if the electron in the conductors is a wave, unlike a particle, it can be in the two parallel branches at the same time, and thus "feel" the magnetic field between these branches.

FIG. 1. A person-electron analogy of the AB effect. 
at hand, and he started to use this knowledge. This may have been the impetus for a correct source analog: a mechanical wave (corresponding to the electron wave) that encounters an obstacle (corresponding to the magnetic field).

452. Ran: Listen, in my opinion it's the only explanation that ..., it's the same electron.

At first Ran (452) did not follow Gal. He seemed to be satisfied with the analogical conclusion (the electron is in the two parallel branches simultaneously, allowing it to "feel" the magnetic field between these branches).

453. Gal: What, like moving, you want to say in fact, well, if it was a wave that propagates? So you say that even if it doesn't really move through there, because it cannot [there is an obstacle], maybe it feels its propagation? Like water, let's take water, when there is a wave, and there is a stone, it sort of bypasses it? But...

454. Ran: It [the water] is continuing to propagate, but it knows that there is a stone there...

455. Gal: So how come, when it bypasses [the stone], it goes back to being a wave...

456. Ran: It doesn't exactly go back. It is changed a bit. This is the special thing. The wave doesn't go back to being the same wave. If there is a stone, a BIG stone, the wave will be diffracted and it will go for instance this way, one toward the other, they will cut one another.

457. Gal: And there will be an interference pattern...

458. Ran: That's it. We'll get interference. This is the wave thing...

Gal's reaction (453) was an elaboration and an explanation of his anchoring example. He explicitly described his problem in the target analog-how the electron is affected by the magnetic field, in terms of the source analog - the mechanical wave that is affected by an obstacle. Gal also generated a concrete example for this description: a water wave that encounters a stone. This time Ran (454) followed Gal's reasoning and treated the water as an agency that "knows" that there is a stone in its path. When Gal (455) explained his difficulty understanding how the stone affects the water, Ran (456) tried to help Gal and started to use his own prior knowledge: he acknowledged that after the wave passes the stone it does not look the same as it did before doing so; he used a concrete description of a water wave that passes a big stone, and he correctly used the formal term "diffraction" in this context. Gal (457) then expressed Ran's description in a more concise scientific terminology of waves, saying that after a water wave bypasses the stone the interference pattern can be seen, and Ran (458) agreed. In our view both students benefited from the exchange. Without Gal's prompting, Ran would not have started to think about the analogical explanation critically. But after Ran started to think about it, he contributed to Gal's understanding by helping Gal to consolidate and explicate his ideas.

459. Gal: So it's actually changing the phase, this magnetic field here? Via,....

460. Ran: How exactly it changes [the phase] I don't know, but it changes [the phase] somehow...

Gal (459) brought the discussion back from the analog source to the analog target. He used the model of mechanical waves that they both elaborated in the source domain to try to explain the electron's behavior (target domain). He said that the magnetic field changes the electron-wave phase. This explanation presents a conceptual development, since Gal replaced the agentive explanation (the electron "feels") with a more mechanistic explanation. Note that the lecturer mentioned that the magnetic field affects the phase of the electron wave, and described the oscillations of the resulting current as an interference pattern. However, the students' recollection and use of these features occurred only at this point in the discussion. Gal, who now understood that the relative phases of the electron waves are affected by the magnetic field, could rephrase his causal puzzle in formal scientific terms, asking how the magnetic field affects this relative phase (in line 463 below he explicitly describes the magnetic field as the corresponding analog to an obstacle in the path of a mechanical wave). Our interpretation of a "missing mechanism" is also backed by Gal's later reflection on his difficulty "It's a force that we simply didn't learn about. This is the problem" (Gal, line 610). A force is the mechanism by which one entity affects another entity in classical physics. Ran (460) shared Gal's mechanistic description. He acknowledged that they formed a causal relation but they lacked the mechanism that could account for this causal relation. It should be noted that in the class $(N=7)$ discussion that followed, Ran and Gal together were able to present, explain, and convince their classmates of their conclusion. Moreover, the excerpt below (463-466) demonstrates that the guided discussion helped Ran and Gal gradually move from viewing the electron as a particle to viewing the electron as a wave.

463. Gal: So all this phase it's.... This [the magnetic field] is like its obstacle?

464. Ran: The electron is not an electron. It's a wave. It's not a particle. It's really...

465. Gal: An electron wave.

466. Ran: A propagating wave and in the middle of this wave there's an obstacle.

In our view, lines 451-466 demonstrate conceptual progress. Gal and Ran gradually formulate a description of the phenomenon ( $\mathrm{AB}$ effect) using relevant formal scientific entities they had previously learned. Neither of them presented this representation at first. This alone could 
be considered clear progress in their knowledge by some researchers [34]. Through the discussion Gal and Ran are able to formulate a causal relation in scientific terms and identify the missing causal mechanism using the appropriate scientific concepts (precisely how the magnetic field changes the phase of the electron wave). This is not a trivial achievement. These students localized an issue in a very specific way, were able to recognize a well-defined gap in their understanding, and explicitly identify the "black box" in the explanation from the lecture. This can be considered a critical step towards understanding, which they were later able to successfully present and defend to their peers. Moreover, Ran and Gal's initial discourse (445-450) suggests that even after the lecture they viewed the electron as a particle, whereas as the discussion progressed their perspective gradually shifted towards a wave description (463-466).

Note that this public web lecture was directed toward 11th and 12th grade students. The lecturer knew that he could not present the formal scientific explanation (a causal mechanism) for the $\mathrm{AB}$ effect since his audiences did not have enough background. He explicitly told his audiences that he was only trying to give them a sense of understanding through the analogy. However, the above episodes show that Gal and Ran fully understood the "black box" only during the discussion; hence the discussion actively fostered their understanding.

The other students only noticed the "black box" during the class discussion when Gal and Ran presented their "case." They started by jointly presenting Ran's difficulty in grasping the "connection" mechanism between the electron's "parts" in both branches of the loop. They used the water-stone analog that they developed for this purpose. Three other students told them repeatedly that in their view there was no problem saying that "it is the same electron in both sides of the loop." However, when Gal and Ran were finally successful in explaining Gal's difficulty, using the terminology of phase difference, and asked how a magnetic field can shift the phase of the electron wave (a question that cannot be answered with high school level prior knowledge in physics), silence prevailed around the table. Gal grinned: "Speechless ha..?" Two of the authors were present in the room at that time, one as the facilitator of the discussion and the other as an observer. Both interpreted this particular silence as conviction and understanding. Gal apparently interpreted this silence in the same manner. Watching this video again during the analysis only reinforced this feeling.

\section{(c) Inventing an alternative causal mechanism}

Ran was willing to accept the "black box" as the missing causal mechanism, but Gal was not satisfied, and he returned to this issue in a later part of the discussion where he generated an alternative causal mechanism. Since Ran did not contribute to this elaboration, only Gal's lines are presented in the following excerpt.
475. Gal: Yes, it propagates like a wave, and then in the middle it has this magnetic field

477. Gal: So it kind of goes out of the conductor. This doesn't make sense...

479. Gal: Let's invent a new theory...

481. Gal: So briefly, yes. There is no other explanation. Just... [silence], yes.

483. Gal: I'm telling you, this wave goes out of the conductor.

485. Gal: There is no other way

491. Gal: It propagates in all the possible paths

Gal assumed that if the electron in the device is indeed a wave, it can propagate in all possible paths (lecture-based idea), thus "part" of it could leave the conductor. This part, small as it may be, could have been affected by the field and be responsible for the phase shift. This inference resolves the inconsistency with the locality of interactions (i.e., Lorentz force). The only causal mechanism that Gal has learned about is force, and he is aware that his alternative mechanism is not complete: "It's a force that we simply didn't learn about" (Gal, line 610). However, he apparently attributes significant explanatory power to his alternative mechanism (485), perhaps because it resonated with his intuitive notion of the locality of interaction, which is well backed by his prior knowledge of science. Our interpretation is also strengthened by Gal's response to the same question a year later. When asked on the delayed posttest, "Why are the electrons in the conducting loop affected by the magnetic field that is in the center of the loop?," he wrote, "When the particle moves as a wave, it spreads everywhere. Therefore it also goes through the place where the magnetic field exists." Another student, Galit, from a different pair developed a similar idea too, but was far less committed to it than Gal and dismissed it during the class discussion that followed. Only Gal presented this idea in the delayed posttest.

\section{B. Tests}

Group 1 completed the full testing sequence on lecture A (quantum mechanics) and group 2 on lecture B (astrophysics). Figure 2 presents quite similar learning curves for both groups on these different lectures. It is clear that prior to the intervention the students knew hardly anything about the subjects presented in the lectures. They performed better just after watching and summarizing a lecture, with a noticeable improvement after the follow-up collaborative activity. Note that the repeated testing was not the main reason for the improvement in the scores after the collaborative session. The content of one question (see the Appendix, lecture B) was not discussed at all during this session. In contrast to the answers for the other questions, we did not see any improvement in the answer to this question in the poststudying test relative to the previously taken postwatching I test $\left(M_{\text {watching } \mathrm{I}}=62.9 \approx\right.$ $M_{\text {studying }}=60.7$ ). 


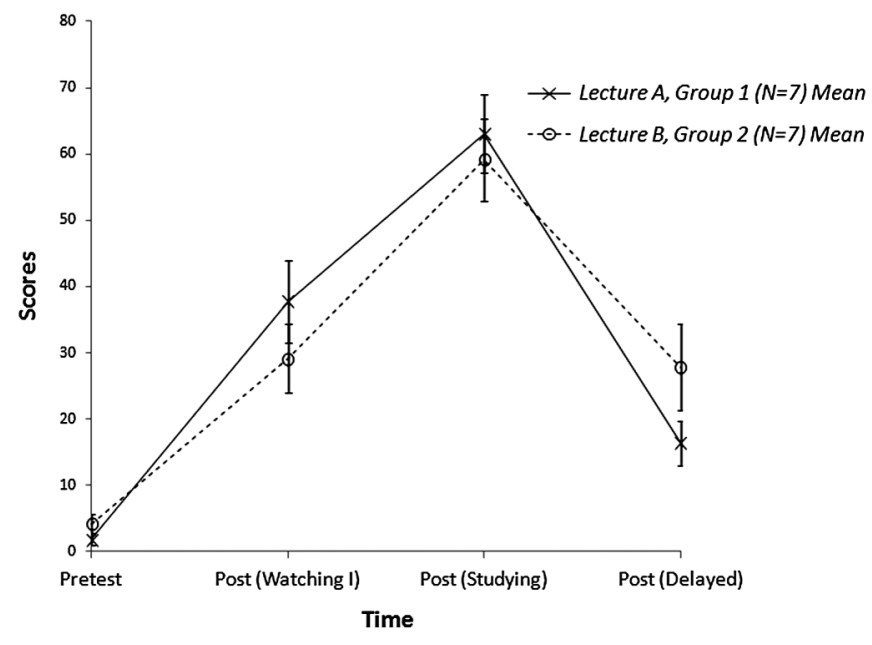

FIG. 2. The average content test scores of both lectures versus time.

The scientific content tests on both lectures were administered to both groups prior to the intervention and a year later. Note that although both groups took the tests on both lectures, each group did the follow-up collaborative activity only on one lecture: group on lecture A and group 2 on lecture B. Figure 3 shows that after a year each group performed much better on the lecture that was processed in the collaborative session, in contrast to the negligible recall from the lecture that was only watched and summarized. The findings indicate that the collaborative session had a noticeable effect on the long-term retention from both lectures.

We hoped that the collaborative follow-up activity would not only help the participants better understand the lecture that was processed, but also teach them to process a public scientific lecture by themselves. Hence we were hoping that although the second lecture was not followed by a collaborative session, the students would perform better on the content knowledge test administered just after watching the second lecture than they had performed on the content test administered just after watching the first

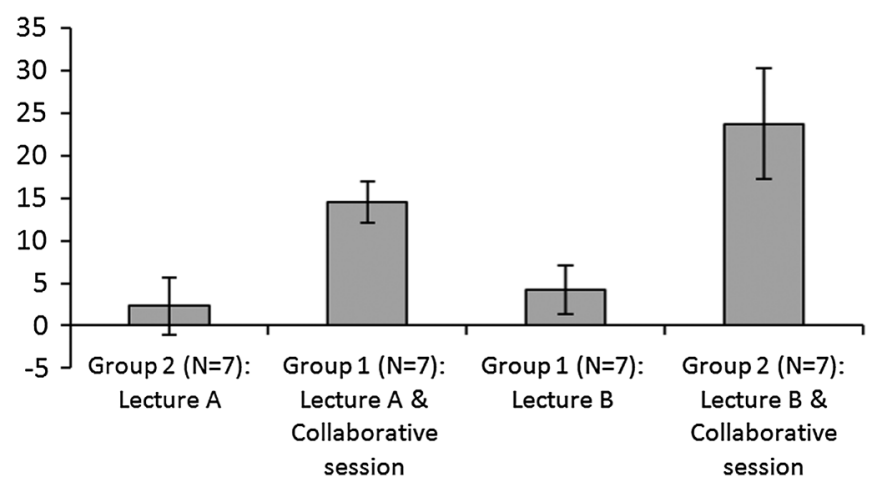

FIG. 3. Long-term retention of content. Average post-delayed test scores relative to the average corresponding pretest scores in the different experimental conditions. lecture (before the collaborative session). The corresponding test results show that this transfer did not take place. One possible explanation for this lack of transfer is that practicing on only one lecture was not sufficient training to acquire new skills. Another explanation could be that this kind of critical processing occurs after a lecture and requires additional time for reflection and thought. Since we did not allocate time for reflection and thought after the second lecture, this could be why we did not see this transfer take place.

\section{SUMMARY AND DISCUSSION}

We described a case study drawn from a teaching experiment where two public physics lectures on advanced topics in physics (astrophysics and quantum mechanics) were utilized to introduce contemporary physics to learners with a high school background in physics. Collaborative mediating activities were designed to scaffold the learning from the lectures.

The scientific content test scores on both lectures show that immediately after they watched a lecture the students knew more about the scientific content presented in this lecture. However, if the lecture was not discussed in the follow-up activity, after a year most of this content was forgotten. The collaborative learning session, which included discussions dealing with critical reformulations of central arguments and analogical explanations, significantly contributed to the students' long-term retention of the content presented in each lecture. This improvement exceeded the improvement expected from repeated testing.

Although we also expected a transfer of skills, namely, that the students would be able to spontaneously derive more knowledge from the second lecture (e.g., with no follow-up activity), we did not observe such a transfer. We are not sure how to explain this. One possibility is that more practice is needed for this transfer to happen. Another possibility is that the critical thinking that we saw in the discussion can only occur when the students spend time thinking about the lecture after they have heard it.

The students' discussions in the context of the follow-up activities clearly illustrate the phenomenon of "going beyond the information given" [35]. Gal and Ran were not just "entertained" by the popular scientific explanation. Their discussion clearly reveals progress: they gradually (1) related what they heard to their prior knowledge, (2) described new knowledge using previously learned abstract scientific terms, (3) employed a new perspective, (4) critiqued the explanation, and (5) identified the causal mechanism that was missing from the causal relation (i.e., the black box). This progress took place during the guided discussion and did not derive solely from the lecture.

Some physics educators would argue that the misconception that Gal developed during the activity is evidence that teaching advanced scientific topics in a popular 
manner is a poor choice since it is bound to produce misconceptions due to gaps in requisite prior knowledge. A study [36] that examined biomedical students' advanced knowledge acquisition identified eight different ways that analogies can induce misconceptions. One is very relevant to this paper. This study classified a group of analogies as very effective at characterizing surface features and relationships but as glossing over underlying causal mechanisms. The findings showed that learners tended either to fill in a convenient but incorrect causal account of their own, or simply left the causal mechanism unexplained, as a kind of black box. Thus, Ran's reaction could be interpreted as settling for the "black box" and Gal's reaction as taking the "tendency to fill in a convenient but incorrect causal account." Although these authors consider the latter tendency negatively in the context of science education, this may not always hold true.

Let us examine the "black box" idea first. After the discussion, Ran and Gal concluded that the magnetic field affects the phase of the electron wave. They consciously defined a black box as the causal mechanism by which this effect occurs. This was done by identifying, narrowing, and isolating what they knew from what they did not know. This is significant evidence of understanding, and a powerful metaconceptual move in learning. It may also promote the efficacy of self-explanations [37].

Gal's "fill in the convenient but incorrect causal account" can also be considered positively. When faced with the missing causal mechanism, Gal incorrectly elaborated the intuitive notion of locality of interaction. However, his alternative causal mechanism should not hinder his understanding of the $\mathrm{AB}$ effect if he ever studies it in a formal manner. According to the formal explanation of the effect, the relevant variable that causes the phase shift is the magnetic vector potential from which the magnetic field is derived. High school students are not familiar with the concept of magnetic vector potential. Although the magnetic field is localized in the AB device, the magnetic vector potential from which it is derived is spread all over the space. Therefore, if Gal ever learns the concept of vector potential, the same intuitive idea that led to his alternative hypothesis (locality of interaction) could be used to support the scientific explanation, strengthening its sense of plausibility.

\section{Implications}

It is worth stressing that utilizing public scientific lectures on contemporary physics topics as an instructional resource cannot and should not replace the traditional formal presentation of classical physics. However, based on our findings, we suggest that a careful exposure to public scientific lectures on contemporary physics topics, accompanied by follow-up activities that process the lecture, in addition and in connection to the formal instruction of classical physics [6], can facilitate the incorporation of contemporary physics into the mainstream curriculum and can present physics in a more relevant and timely light. In fact, we believe that the generic activities that we used in this study could help utilize other popular presentations of science in the media such as TV shows and popular science texts, or be used as a follow-up activity after a live public lecture that the class is attending out of school. Moreover, since most high school students will not be physicists or even scientists in the future, educating them to learn science from popular science resources is an important goal for lifelong learning.

\section{APPENDIX: CONTENT KNOWLEDGE TESTS}

See separate auxiliary material for the knowledge tests, summary of the lecture, summary of activity, and worksheet.
[1] The Perimeter Institute Of Theoretical Physics Public Lectures, http://www.perimeterinstitute.ca/en/outreach/ public_lectures/view_past_public_lectures/.

[2] Space Telescope Science Institute Public Lecture Series, https://webcast.stsci.edu/webcast/searchresults .xhtml searchtype $=20 \&$ eventid $=40 \&$ sortmode $=1$.

[3] L. J. Rennie and G. F. Williams, The nature and measurement of learning from a lecture series in human genetics, Melbourne Stud. Educ. 41, 17 (2000).

[4] S. Kapon, U. Ganiel, and B. Eylon, Goals and design of public physics lectures: perspectives of high-school students, physics teachers and lecturers, Phys. Educ. 44, 528 (2009).

[5] S. Kapon, U. Ganiel, and B. Eylon, in Proceedings of the Physics Education Research Conference, Greensboro, NC,
2007, edited by L. Hsu, C. Henderson, and L. Mccullough (AIP, New York, 2007), pp. 124-127.

[6] S. Kapon, U. Ganiel, and B. Eylon, Scientific argumentation in public physics lectures: bringing contemporary physics into high school teaching, Phys. Educ. 44, 33 (2009).

[7] S. Kapon, U. Ganiel, and B. Eylon, Explaining the unexplainable: Translated scientific explanations (TSE) in public physics lectures, Int. J. Sci. Educ. 32, 245 (2010).

[8] C. H. Crouch and E. Mazur, Peer instruction: Ten years of experience and results, Am. J. Phys. 69, 970 (2001).

[9] R. R. Hake, arXiv:physics/0106087.

[10] E.F. Redish, Teaching Physics: With the Physics Suite (Wiley, Hoboken, NJ, 2003), Vol. xi, p. 216. 
[11] F. J. Di Vesta and G. S. Gray, Listening and note taking, J. Educ. Psychol. 63, 8 (1972).

[12] B. M. Frank, Effect of field independence-dependence and study technique on learning from a lecture, Am. Educ. Res. J. 21, 669 (1984).

[13] K. A. Kiewra, A review of note-taking: The encodingstorage paradigm and beyond, Educ. Psychol. Rev. 1, 147 (1989).

[14] A. King, Comparison of self-questioning, summarizing, and notetaking-review as strategies for learning from lectures, Am. Educ. Res. J. 29, 303 (1992).

[15] Z. Hrepic, D. A. Zollman, and S. N. Rebello, Comparing students' and experts' understanding of the content of a lecture, J. Sci. Educ. Tech. 16, 213 (2007).

[16] A. Zohar and F. Nemet, Fostering students' knowledge and argumentation skills through dilemmas in human genetics, J. Res. Sci. Teach. 39, 35 (2002).

[17] C. von Aufschnaiter et al., Arguing to learn and learning to argue: Case studies of how students' argumentation relates to their scientific knowledge, J. Res. Sci. Teach. 45, 101 (2008).

[18] M. C. Linn, B.S. Eylon, and E. A. Davis, in Internet Environments for Science Education, edited by M.C. Linn, E. A. Davis, and P. B. Bell (Erlbaum, Mahwah, NJ, 2004), pp. 29-46.

[19] P. Bell and M. C. Linn, Scientific arguments as learning artifacts: Designing for learning from the web with KIE, Int. J. Sci. Educ. 22, 797 (2000).

[20] N. Miyake and H. Shirouzu, in Proceedings of the Sixth International Conference on Learning Sciences, Santa Monica, CA, 2004 (Lawrence Erlbaum, Hillsdale, NJ, 2004).

[21] G. R. Funkhouser and N. Maccoby, Tailoring science writing to the general audience, Journalism Q. 50, 220 (1973).

[22] L. D. Yore, B. M. Hand, and M. K. Florence, Scientists' views of science, models of writing, and science writing practices, J. Res. Sci. Teach. 41, 338 (2004).

[23] J. Clement, Observed methods for generating analogies in scientific problem solving, Cogn. Sci. 12, 563 (1988).
[24] J. Clement, Using bridging analogies and anchoring intuitions to deal with students' preconceptions in physics, J. Res. Sci. Teach. 30, 1241 (1993).

[25] J. Clement, Expert novice similarities and instruction using analogies, Int. J. Sci. Educ. 20, 1271 (1998).

[26] Z.R. Dagher, Review of studies on the effectiveness of instructional analogies in science education, Sci. Educ. 79, 295 (1995).

[27] A. Harrison and D. Treagust, in Metaphor and Analogy in Science Education, edited by P.J. Aubusson, A.G. Harrospn, and S. M. Ritchie (Springer, Dordrecht, 2006), pp. 11-24.

[28] R. K. Yerrick et al., Social interaction and the use of analogy: An analysis of preservice teachers' talk during physics inquiry lessons, J. Res. Sci. Teach. 40, 443 (2003).

[29] S. M. Glynn et al., in Handbook of Creativity, edited by J. A. Glover, R. R. Ronning, and C. R. Reynolds (Plenum, New York, 1989), pp. 383-398.

[30] P. Thagard, Analogy, explanation, and education, J. Res. Sci. Teach. 29, 537 (1992).

[31] E.D. Wong, Understanding the generative capacity of analogies as a tool for explanation, J. Res. Sci. Teach. 30, 1259 (1993).

[32] Y. Aharonov and D. Bohm, Further considerations on electromagnetic potentials in the quantum theory, Phys. Rev. 123, 1511 (1961).

[33] R. P. Feynman, R. B. Leighton, and M. Sands, The Feynman Lectures on Physics (Addison Wesley, Reading, MA, 1971), Vol. 2.

[34] M. T. H. Chi and S. Ohlsson, in The Cambridge Handbook of Thinking and Reasoning, edited by K. J. Holyoak and R. G. Morrison (Cambridge University Press, Cambridge, England, 2005), pp. 371-399.

[35] J.S. Bruner, in Beyond the Information Given: Studies in the Psychology of Knowing, edited by J. S. Bruner (George Allen \& Unwin, London, 1974), pp. 218-238.

[36] R. J. Spiro et al., in Similarity and Analogical Reasoning, edited by S. Vosniadou and A. Ortony (Cambridge University Press, New York, 1989), pp. 498-531.

[37] M. T.H. Chi et al., Eliciting self-explanations improves understanding, Cogn. Sci. 18, 439 (1994). 